\title{
Juvenile idiopathic arthritis complicated by amyloidosis with secondary nephrotic syndrome - effective treatment with tocilizumab
}

\author{
Małgorzata Kwiatkowska, Ewa Jednacz, Lidia Rutkowska-Sak
}

Department and Polyclinic of Developmental Age Rheumatology, Institute of Rheumatology, Warsaw, Poland

\begin{abstract}
A case report of a boy with juvenile idiopathic arthritis since the age of 2 years, generalized onset, complicated by nephrotic syndrome due to secondary type A amyloidosis is presented. In the patient the disease had an especially severe course, complicated by frequent infections, making routine treatment difficult. Amyloidosis was diagnosed in the $5^{\text {th }}$ year of the disease based on a rectal biopsy. Since the disease onset the boy has been taking prednisolone and sequentially cyclosporine A, methotrexate, chlorambucil, etanercept, and cyclophosphamide. Clinical and laboratory remission was observed after treatment with tocilizumab. After 42 months of treatment with tocilizumab the boy's condition is good. There is no pain or joint edema, and no signs of nephrotic syndrome.
\end{abstract}

Key words: juvenile idiopathic arthritis, amyloidosis, tocilizumab.

Juvenile idiopathic arthritis (JIA) is the most common systemic inflammatory disease of the connective tissue at the developmental age.

Generalized juvenile idiopathic arthritis $(g / A)$ is observed in up to $20 \%$ of children with arthritis. Organ-associated symptoms are typical for this form of the disease: recurrent fever lasting for at least 2 weeks, rash of different forms (exacerbating with a body temperature increase), serositis, enlarged liver and/or spleen, and enlarged lymph nodes. Arthritis can be present since the onset, but it is not an obligatory condition to diagnose this disease. With regard to further disease evolution the intensity is various and it can involve several or many joints; additionally, symptoms of internal organ involvement may be predominant.

Laboratory test results reveal elevated inflammatory markers, anemia, and elevated white blood cell and platelet counts, which often persist despite treatment with glucocorticosteroids and disease-modifying antirheumatic drugs (DMARDs).
Amyloidosis, extracellular accumulation of amyloid A, leading to multiple organ failure, especially kidney and liver failure, is a serious complication of gJIA.

\section{Case report}

The case concerns a 16-year-old boy who has suffered from gJIA since the age of 2 years. A rapid onset of the disease was observed - fever up to $40^{\circ} \mathrm{C}$, transient rashes, enlarged liver, spleen and lymph nodes, since the $6^{\text {th }}$ week of the disease inflammation of the knee joints, and 2 weeks later, of the ankles. The symptoms resulted in significantly impaired independent mobility of the child.

At the beginning, when prolonged infection was suspected, the boy was treated with antibiotics and non-steroid anti-inflammatory agents in an outpatient setting. Due to exacerbation of the child's condition, persistent general symptoms and arthritis, the child was hospitalized at a regional hospital and a diagnosis of 
gIIA was made based on symptoms reported and when other causes were excluded.

Treatment included intravenous glucocorticosteroids at a dose of $3 \mathrm{mg} / \mathrm{kg} / 24 \mathrm{~h}$ and improvement was observed - the body temperature normalized, and pain and joint edema decreased. When the medicine dose was gradually and extremely slowly reduced and then replaced with an oral form, general symptoms returned but transiently. After 9 months of the disease treatment was additionally supplemented with cyclosporine A at a dose of $3 \mathrm{mg} / \mathrm{kg} / 24 \mathrm{~h}$ and the boy was transferred to the supervision of the Department and Polyclinic of Developmental Age Rheumatology, Institute of Rheumatology, Warsaw. Previous treatment was continued and the dose of glucocorticosteroids was reduced extremely slowly, and one year later, due to disease exacerbation (inflammation of the ankles, knee joints, wrists and elbows returned) treatment was additionally supplemented with methotrexate.

As early as in the first year of the disease despite supplementation with vitamin D3 and calcium there was a compressive fracture of the lumbar spine ( $L 4)$, and in the following years of the $L 3$ vertebral body and thoracic vertebrae (Th6-Th11).

Due to recurrent severe respiratory tract infections (pneumonia) within the first 3 years of the disease combined with respiratory failure (temporary intubation was necessary twice), immunosuppressive therapy was not regular, and the underlying disease was constantly active.

Apart from joints that were already involved, inflammation of the small joints in the hands (MCP, PIP), shoulders and hips as well as pain in the cervical spine combined with progressive mobility limitation was observed.

Due to the fact that proteinuria had increased since the $4^{\text {th }}$ year of the disease, there was hepatomegaly and splenomegaly, and elevated inflammatory markers were still observed, in the $5^{\text {th }}$ year a biopsy of the colon mucosa was performed.

Table I. Treatment used in the reported patient

\begin{tabular}{|lc|}
\hline Treatment & \multicolumn{1}{c|}{ Period } \\
\hline Glucocorticosteroids & Jan 2000 - present \\
\hline Cyclosporine A & Sep 2000 - Oct 2004 \\
\hline Methotrexate & $\begin{array}{c}\text { Aug 2001 - Sep 2004 } \\
\text { Feb 2012 - present }\end{array}$ \\
\hline Chlorambucil & Oct 2004 - Mar 2011 \\
\hline Etanercept & Feb 2005 - Mar 2011 \\
\hline Cyclophosphamide & May 2011 - Oct 2011 \\
\hline Tocilizumab & Nov 2011 - present \\
\hline
\end{tabular}

Histopathology revealed presence of amyloid deposits. At that time treatment included chlorambucil, and methotrexate and glucocorticoids were continued. Treatment with cyclosporine A was completed (Table I). Despite modification of pharmacotherapy, clinical or laboratory improvement was not observed.

In the $6^{\text {th }}$ year the boy was considered eligible for biological treatment with etanercept and was taking this agent for 6 years. Complete remission was not achieved, but joint swelling decreased, and laboratory inflammatory markers slightly decreased. Biological treatment was administered irregularly due to a difficult economic situation of the child's family and problems transporting the child to the rheumatology clinic. During longer intervals lasting several weeks when etanercept was not administered, disease exacerbation was observed - pain and swelling of the joints, no fever or rash, moderately elevated inflammatory markers and normal results of liver and kidney function tests. During treatment with etanercept and chlorambucil, proteinuria up to $1.2 \mathrm{~g} / 24 \mathrm{~h}$ was periodically observed but without signs of nephrotic syndrome.

In the $10^{\text {th }}$ year of the disease (in 2009, the patient was 12 years old) surgery on soft tissues was performed to release contraction of the hip joints (release of pathologically contracted proximal attachment of the sartorius muscle, rectus femoris muscle, iliopsoas muscle and tensor fasciae latae).

In the $12^{\text {th }}$ year of the disease after mild herpes zoster infection the child's condition suddenly deteriorated - pain and swelling developed, and mobility of almost all joints in the lower and upper extremities was limited to a higher degree than observed in the past, with minimum mobility of the cervical spine. Apart from joint edema there was massive edema of the feet and lower legs, the liver was enlarged and reaching the iliac ala, and there was tachycardia and tachypnea.

Laboratory test results revealed elevated inflammatory markers: $\mathrm{CRP}=29 \mathrm{mg} / \mathrm{dl}$ ( $\mathrm{n}$. to 10.0), $\mathrm{ESR}=108$ $\mathrm{mm} / \mathrm{h}$, anemia with thrombocytemia, significant decrease in the total protein serum levels to $4.1 \mathrm{~g} / \mathrm{dl}$ with albumin levels reduced to below $1.5 \mathrm{~d} / \mathrm{dl}$, proteinuria up to $40 \mathrm{~g} / 24 \mathrm{~h}$, creatinine and urea blood serum levels within the norm, aminotransferase activity within the norm, and activity of $\gamma$-glutamyltranspeptidase elevated 3-fold. A kidney biopsy was performed - segmental hyalinization of all glomeruli was demonstrated. In the parenchyma there was scarce infiltration of mononuclear cells, and few tubules with dilated lumens. The test for amyloid A was positive.

Treatment included intravenous cyclophosphamide infusions - at a dose of $20 \mathrm{mg} / \mathrm{kg}$ bw - once a month 
for 5 successive months, and 20\% albumin was administered intravenously several times.

Proteinuria decreased to $18 \mathrm{~g} / 24 \mathrm{~h}$, but symptoms of nephrotic syndrome were still present. Tocilizumab at a dose of $8 \mathrm{mg} / \mathrm{kg}$ every two weeks was started. The child's condition slowly but gradually improved, especially with regard to the joints - pain and edema decreased, and the range of motion increased.

After the $10^{\text {th }}$ dose of tocilizumab inflammatory markers normalized. Proteinuria gradually decreased as early as after the first dose of tocilizumab (18 $\rightarrow 8 \mathrm{~g} / 24 \mathrm{~h})$, and from the $15^{\text {th }}$ dose it was below $1.0 \mathrm{~g} / 24 \mathrm{~h}$.

After 12 months of treatment with tocilizumab the boy had respiratory tract infection complicated by bronchogenic pneumonia. He did not report for a scheduled tocilizumab infusion, and it resulted in disease exacerbation - pain and edema of several joints returned and inflammatory markers were elevated once again: $\mathrm{ESR}=93 \mathrm{~mm} / \mathrm{h}, \mathrm{CRP}=22 \mathrm{mg} / \mathrm{dl}$ (norm up to 10), but daily proteinuria did not increase (0.2-0.35 g/24 h).

After a prescribed dose of tocilizumab $(240 \mathrm{mg})$ the child's condition rapidly improved, and follow-up blood tests performed 2 weeks later revealed normalization of inflammatory markers.

After 42 months of treatment with tocilizumab the boy's condition is good. There is no pain or joint edema, no signs of nephrotic syndrome, the liver is of a normal size, laboratory test results are within the norm, and daily proteinuria is below $0.25 \mathrm{~g} / 24 \mathrm{~h}$ (no proteinuria in random tests).

A follow-up abdominal ultrasound revealed that the liver was not enlarged, with normal echogenicity.

The patient will continue to receive tocilizumab every 2 weeks.

\section{Discussion}

Generalized juvenile idiopathic arthritis is the most severe subtype, differing from other subtypes not only with regard to a clinical course but also with regard to its pathogenesis. Currently, it is thought to be an autoinflammatory disease, contrary to the autoimmune etiology of other JIA subtypes [1]; however, some authors claim that enthesitis-related arthritis (ERA) also has autoinflammatory background. It is obvious that cytokines play a role in the pathogenesis, and (interleukin- 6 IL-6) plays a key role in systemic JIA - it is a pleiotropic cytokine with a wide spectrum of biological activity, participating in immune responses, inflammatory reactions and hematopoiesis. High levels of (IL-6) were observed in the blood, articular fluid and synovial membrane in patients with rheumatoid arthritis and in children with systemic JIA [2]. The IL-6 levels correlate with disease ac- tivity and increased levels of acute phase proteins and thrombocytosis.

Amyloidosis is most frequently observed in children with systemic JIA. The IL-6-induced acute phase proteins include serum amyloid A (SAA) that causes not only secondary amyloidosis but also stimulates rheumatoid fibroblast-like synoviocytes (FLS) to produce IL-6 [3].

There have been reports on the effective treatment of amyloidosis due to rheumatoid diseases with TNF antagonists. It is thought that their mechanism of action consists in controlling the inflammatory condition leading to reduced SAA levels, as serum amyloid precursor protein is produced in the liver as an acute phase protein $[1,2]$.

Tocilizumab is a humanized monoclonal antibody targeted at human IL- 6 receptors. It selectively binds to membrane-bound and soluble IL-6 receptors, resulting in the inhibition of its biological properties. Tocilizumab has proven to be effective in rheumatoid arthritis and in other systemic diseases of the connective tissue [4,5].

There are more and more reports confirming the efficacy of tocilizumab treatment in the case of systemic JIA not responding to standard treatment [6-8], but there are only a few reports regarding beneficial effects of tocilizumab on amyloidosis in JIA [6], especially with regard to the treatment of nephrotic syndrome $[9,10]$.

Spanish authors have published a paper on beneficial effects of tocilizumab on the course of nephrotic syndrome in a patient with rapidly progressing amyloidosis secondary to the latent form of tuberculosis [11].

There have been several papers on the efficacy of tocilizumab with regard to gastrointestinal symptoms due to intestinal amyloidosis due to RA [8, 12-14].

The use of tocilizumab does not seem to be associated with an increased risk of macrophage activation syndrome in the course of systemic JIA [15].

In the reported patient the disease had an especially severe course, complicated by frequent infections, making routine treatment difficult. Amyloidosis was diagnosed in the 5th year of the disease based on a rectal biopsy. Since the disease onset the boy has been taking DMARDs and prednisolone. In the $7^{\text {th }}$ year biological treatment was started and the child's condition improved, and inflammatory markers decreased. It seems that treatment with etanercept inhibited or significantly slowed down the course of amyloidosis as proteinuria did not increase within 5 years of treatment. A turning point was herpes zoster infection, as later the boy developed nephrotic syndrome, and a kidney biopsy confirmed amyloidosis, apart from visible segmental lesions in all glomeruli, similarly as in the case of hyalinization. Due to unsatisfactory improvement following cyclophosphamide infusions, we decided to start treatment with tocilizumab, and consequently the child's condition 
slowly improved and proteinuria regressed. As it was possible to achieve remission of nephrotic syndrome and systemic JIA, we can conclude that treatment with tocilizumab may be an important option of treatment of secondary amyloidosis that develops in the course of rheumatic diseases.

The authors declare no conflict of interest.

\section{References}

1. Sikora KA, Grom AA. Update on the pathogenesis and treatment of systemic idiopathic arthritis. Curr Opin Pediatr 2011; 23: 640-646.

2. Martini A. Systemic juvenile idiopathic arthritis. Autoimmun Rev 2012; 12: 56-59.

3. Koga T, Torigoshi T, Motokawa S, et al. Serum amyloid A-induced IL- 6 production by rheumatoid synoviocytes. FEBS Lett 2008; 582: 579-585.

4. De Benedetti F, Brunner HI, Ruperto N, et al. Randomized trial of tocilizumab in systemic juvenile idiopathic arthritis. N Engl J Med 2012; 3637: 2385-2395.

5. Brunner H, Ruperto N, Zuber Z, et al. A4: Efficacy and safety of tocilizumab in patients with polyarticular-coursejuvenile idiopathic arthritis: 2-year data from CHERISH. Arthritis Rheum 2014; 66 Suppl 11: S5-6.

6. Okuda Y, Takasugi K. Successful use of a humanized anti-interleukin-6 receptor antibody, tocilizumab, to treat amyloid A amyloidosis complicating juvenile idiopathic arthritis. Arthritis Rheum 2006; 54: 2997-3000.

7. Okuda Y, Ohnishi M, Matoba K, et al. Comparison of the clinical utility of tocilizumab and anti-TNF therapy in AA amyloidosis complicating rheumatic diseases. Mod Rheumatol 2014; 24: 137-143.

8. Matsui M, Okayama S, Tsushima $\mathrm{H}$, et al. Therapeutic benefits of tocilizumab vary in different organs of a patient with AA amyloidosis. Case Rep Nephrol 2014; 2014: ID 823093.

9. Miyagawa I, Nakayamada S, Saito K, et al. Study on the safety and efficacy of tocilizumab, an anti-IL-6 receptor antibody, in patients with rheumatoid arthritis complicated with AA amyloidosis. Mod Rheumatol 2014; 24: 405-409.

10. De La Torre M, Arboleya L, Pozo S, et al. Rapid and sustained response to tocilizumab, anti-interleukin-6 receptor antibody, in a patient with nephrotic syndrome secondary to systemic juvenile idiopathic arthritis-related amyloidosis. NDT Plus 2011; 4: 178-180.

11. Magro-Checa C, Navas-Parejo Casado A, Borrego-García E, et al. Successful use of tocilizumab in a patient with nephrotic syndrome due to a rapidly progressing AA amyloidosis secondary to latent tuberculosis. Amyloid 2011; 18: 235-239.

12. Nishida S, Hagihara K, Shima Y, et al. Rapid improvement of AA amyloidosis with humanised anti-interleukin 6 receptor antibody treatment. Ann Rheum Dis 2009; 68: 1235-1236.

13. Sato H, Sakai T, Sugaya T, et al. Tocilizumab dramatically ameliorated life-threatening diarrhea due to secondary amyloidosis associated with rheumatoid arthritis. Clin Rheumato 2009; 28: 1113-1116.
14. Inoue D, Arima H, Kawanami C, et al. Excellent therapeutic effect of tocilizumab on intestinal amyloid a deposition secondary to active rheumatoid arthritis. Clin Rheumatol 2010; 29: 1195-1197.

15. Ravelli A, Schneider R, Weitzman S, et al. A56: Macrophage activation syndrome in patients with systemic juvenile idiopathic arthritis treated with tocilizumab. Arthritis Rheum 2014; 66 Suppl 11: S83-84. 